\title{
The Genus of the Erdős-Rényi Random Graph and the Fragile Genus Property
}

\section{Chris Dowden ${ }^{1}$}

Institute of Discrete Mathematics, Graz University of Technology, 8010 Graz, Austria

dowden@math.tugraz.at

Mihyun Kang ${ }^{2}$

Institute of Discrete Mathematics, Graz University of Technology, 8010 Graz, Austria kang@math.tugraz.at

\section{Michael Krivelevich ${ }^{3}$}

School of Mathematical Sciences, Sackler Faculty of Exact Sciences, Tel Aviv University, Tel Aviv 6997801, Israel

krivelev@post.tau.ac.il

\begin{abstract}
We investigate the genus $g(n, m)$ of the Erdős-Rényi random graph $G(n, m)$, providing a thorough description of how this relates to the function $m=m(n)$, and finding that there is different behaviour depending on which 'region' $m$ falls into.

Existing results are known for when $m$ is at most $\frac{n}{2}+O\left(n^{2 / 3}\right)$ and when $m$ is at least $\omega\left(n^{1+\frac{1}{j}}\right)$ for $j \in \mathbb{N}$, and so we focus on intermediate cases.

In particular, we show that $g(n, m)=(1+o(1)) \frac{m}{2}$ whp (with high probability) when $n \ll$ $m=n^{1+o(1)}$; that $g(n, m)=(1+o(1)) \mu(\lambda) m$ whp for a given function $\mu(\lambda)$ when $m \sim \lambda n$ for $\lambda>\frac{1}{2}$; and that $g(n, m)=(1+o(1)) \frac{8 s^{3}}{3 n^{2}}$ whp when $m=\frac{n}{2}+s$ for $n^{2 / 3} \ll s \ll n$.

We then also show that the genus of fixed graphs can increase dramatically if a small number of random edges are added. Given any connected graph with bounded maximum degree, we find that the addition of $\epsilon n$ edges will whp result in a graph with genus $\Omega(n)$, even when $\epsilon$ is an arbitrarily small constant! We thus call this the 'fragile genus' property.
\end{abstract}

2012 ACM Subject Classification Mathematics of computing $\rightarrow$ Random graphs, Mathematics of computing $\rightarrow$ Graphs and surfaces

Keywords and phrases Random graphs, Genus, Fragile genus

Digital Object Identifier 10.4230/LIPIcs.AofA.2018.17

Acknowledgements We would like to thank Philipp Sprüssel for helpful discussions, and we are also grateful to the reviewers for their comments.

\section{Introduction}

\subsection{Background and motivation}

The Erdős-Rényi random graph $G(n, m)$ (taken uniformly at random from the set of all labelled graphs with vertex set $[n]=\{1,2, \ldots, n\}$ and exactly $m$ edges) and the binomial

1 Supported by Austrian Science Fund (FWF): P27290.

2 Supported by Austrian Science Fund (FWF): P27290.

3 Supported in part by USA-Israel BSF grant 2014361, and by grant 1261/17 from Israel Science Foundation. 
random graph $G_{n, p}$ (the graph on $[n]$ where every edge occurs independently at random with probability $p$ ) have been a source of fascination for many decades, producing numerous exciting results (see, for example, [3], [5], and [6]).

In this work, we are interested in the genus of a graph. A graph is said to have genus $g$ if this is the minimum number of handles that must be attached to a sphere in order to be able to embed the graph without any crossing edges. Hence, the simplest case when $g=0$ corresponds to planar graphs.

The genus is one of the most fundamental properties of a graph, and plays an important role in a number of applications and algorithms (e.g. colouring problems and the manufacture of electrical circuits). It is naturally intriguing to consider the genus of a random graph, and such matters are also related to random graphs on surfaces (see, for example, Question 8.13 of [7] and Section 9 of [4]).

The genus of the binomial random graph $G_{n, p}$ was first studied in [1], and it was shown that this is $(1+o(1)) \frac{p n^{2}}{12}$ with high probability (whp for short, meaning with probability tending to 1 as $n \rightarrow \infty-$ see Definition 7$)$ if $p^{2}\left(1-p^{2}\right) \geq \frac{8(\log n)^{4}}{n}$. A particularly notable consequence of this result (by taking $p=\frac{1}{2}$ ) is that the classical uniform random graph $G(n)$ (taken uniformly at random from the set of all labelled graphs on $[n]$ ) must then have genus $(1+o(1)) \frac{n^{2}}{24}$ whp.

As noted in [1], results for the genus of $G_{n, p}$ can be transferred into analogous results for the genus $g(n, m)$ of $G(n, m)$. Taking into account the work in both [1] and [16] (the latter of which deals with a substantially wider range for $p)$, these show that $g(n, m)=(1+o(1)) \frac{m}{6}$ whp when $m=\Theta\left(n^{2}\right)$ and that $g(n, m)=(1+o(1)) \frac{j m}{2(j+2)}$ whp when $n^{1+\frac{1}{j+1}} \ll m \ll n^{1+\frac{1}{j}}$ for $j \in \mathbb{N}$.

Separately, important work has also been carried out to determine the probability that $G(n, m)$ is planar (i.e. has zero genus) when $m$ is comparatively small. In particular, it is now well-known that $G(n, m)$ is planar whp when $m<\frac{n}{2}-\omega\left(n^{2 / 3}\right)$ (see [14]) and that $\lim \inf \mathbb{P}\left[G(n, m)\right.$ is planar] $>0$ when $m=\frac{n}{2}+O\left(n^{2 / 3}\right)$ (see [14], and see [15] for exact limiting probabilities).

It is our aim here to now bridge the gap between the $m \gg n^{1+\frac{1}{j+1}}$ and $m=\frac{n}{2}+O\left(n^{2 / 3}\right)$ results. We provide a thorough description of this intermediate region, finding that there is different behaviour depending on whether (i) $n \ll m=n^{1+o(1)}$, (ii) $m \sim \lambda n$ for $\lambda>\frac{1}{2}$, or (iii) $m=\frac{n}{2}+s$ for $s>0$ satisfying $n^{2 / 3} \ll s \ll n$.

We then turn our attention to a related problem, concerning the genus of a graph that is partially random. Here, we take an arbitrary connected graph $H$ with bounded maximum degree, and examine the supergraph $G$ formed by adding some random edges to $H$ (this type of model is sometimes called 'smoothed analysis' or a 'randomly perturbed' graph, see e.g. [2], [8], [9], and [11]).

Rather surprisingly, we find that $G$ will whp have high genus, even if $H$ has low genus and the number of random edges added is relatively small. We thus call this the 'fragile genus' property.

\subsection{Main results and techniques}

The main contributions of this paper are two-fold. Firstly, we obtain a complete picture of $g(n, m)$ for all values of $m$ by producing precise results for the previously uncharted regions. Secondly, we then initiate the study of how the genus of a fixed graph is affected when random edges are added, discovering the fragile genus property. 
We now present our main results in detail. In the first of these, we consider $g(n, m)$ for the region when $n \ll m=n^{1+o(1)}$ (e.g. this would be the case for a function such as $m=n \ln n)$. Note that this is not an area that is covered by existing work. However, we obtain the following tight bounds:

- Theorem 1. Let $m=m(n)$ satisfy $n \ll m=n^{1+o(1)}$. Then with high probability

$$
(1-o(1)) \frac{m}{2} \leq g(n, m) \leq \frac{m}{2} .
$$

Perhaps the most obvious gap in previous knowledge concerns the case when $m$ is linear in $n$, but above the threshold for planarity. We show that the genus behaves smoothly in this region:

- Theorem 2. Let $m(n) \sim \lambda n$ for some fixed $\lambda>\frac{1}{2}$. Then with high probability

$$
g(n, m)=(1+o(1)) \mu(\lambda) m,
$$

where

$$
\mu(\lambda)=\frac{1}{4 \lambda^{2}} \sum_{r=1}^{\infty} \frac{r^{r-2}}{r !}\left(2 \lambda e^{-2 \lambda}\right)^{r}+\frac{1}{2}\left(1-\frac{1}{\lambda}\right)
$$

is a strictly positive, monotonically increasing, continuous function satisfying $\mu(\lambda) \rightarrow 0$ as $\lambda \rightarrow \frac{1}{2}$ and $\mu(\lambda) \rightarrow \frac{1}{2}$ as $\lambda \rightarrow \infty$.

One of the most fascinating areas of study in random graphs has been the behaviour of $G(n, m)$ when $m$ is close to $\frac{n}{2}$, as many important features have been found to emerge around this key point. Here, we examine in detail the slightly supercritical regime when $m=\frac{n}{2}+s$ for $s>0$ satisfying $n^{2 / 3} \ll s \ll n$ (i.e. precisely the region between the planarity threshold and the linear case dealt with in Theorem 2), showing exactly how the genus grows:

- Theorem 3. Let $m(n)=\frac{n}{2}+s(n)$, where $s=s(n)$ satisfies $s>0$ for all $n$ and $n^{2 / 3} \ll$ $s \ll n$. Then with high probability

$$
g(n, m)=(1+o(1)) \frac{8 s^{3}}{3 n^{2}} .
$$

All these results are summarised in Table 1, which gives an exciting picture of how the genus $g=g(n, m)$ behaves as $m$ grows. In particular, it is intriguing to see that the ratio of $g$ to $m$ increases from 0 to $\frac{1}{2}$ until $m$ becomes superlinear in $n$, after which it then decreases from $\frac{1}{2}$ to $\frac{1}{6}$.

Our proofs typically utilise Euler's formula. Given a graph $G$, this states that the genus $g(G)$ satisfies

$$
g(G)=\frac{1}{2}(e(G)-|G|-f(G)+\kappa(G)+1),
$$

where $e(G)$ is the number of edges of $G,|G|$ is the number of vertices of $G, f(G)$ is the number of faces of $G$ when embedded on a surface of minimal genus (i.e. a sphere to which $g(G)$ handles have been attached), and $\kappa(G)$ is the number of components of $G$.

Consequently, our results often involve first establishing new bounds for the number of faces of $G(n, m)$ (for instance, via the number of short cycles).

For the proof of Theorem 3, in order to attain the required level of accuracy, we note that we actually find it better to deal directly with the 2-core of $G(n, m)$, rather than with the entire graph. 
Table 1 A summary of $g:=g(n, m)$.

\begin{tabular}{|l||l||l|}
\hline$m=\Theta\left(n^{2}\right)$ & $g=(1+o(1)) \frac{m}{6}$ whp & See [16] \\
\hline$n^{1+\frac{1}{j+1} \ll m \ll n^{1+\frac{1}{j}}}$ & $g=(1+o(1)) \frac{j m}{2(j+2)}$ whp & See [16] \\
\hline$m=\Theta\left(n^{1+\frac{1}{j}}\right)$ & $(1+o(1)) \frac{(j-1) m}{2(j+1)}$ & See [16] \\
\hline$n \ll m=n^{1+o(1)}$ & $(1-o(1)) \frac{m}{2} \leq g \leq \frac{m}{2}$ whp & Theorem 1 \\
\hline$m \sim \lambda n, \lambda>\frac{1}{2}$ & $g=(1+o(1)) \mu(\lambda) m$ whp, & Theorem 2 \\
\hline$m=\frac{n}{2}+s$, & where $\mu(\lambda) \rightarrow 0$ as $\lambda \rightarrow \frac{1}{2}$ & \\
\hline$s>0$ and $n^{2 / 3} \ll s \ll n$ & and $\mu(\lambda) \rightarrow \frac{1}{2}$ as $\lambda \rightarrow \infty$ & Theorem 3 \\
\hline$m-\frac{n}{2} \sim c n^{2 / 3}$ & $g=(1+o(1)) \frac{8 s^{3}}{3 n^{2}}$ whp & \\
\hline$m<\frac{n}{2}-\omega\left(n^{2 / 3}\right)$ & $\lim n \rightarrow \infty \mathbb{P}(g=0)=r(c) \in(0,1)$, & See [14] \\
\hline where $r(c) \rightarrow 1$ as $c \rightarrow-\infty$ & See [14] \\
\hline
\end{tabular}

We now turn our attention to our final main result, which concerns the fragile genus property. Here, we take an arbitrary connected graph $H$ with bounded maximum degree, and a random graph $R$ on the same vertex set, and we consider the genus $g(G)$ of the graph $G=H \cup R$. We make an interesting discovery, finding that $g(G)$ will whp be rather large, even if $H$ and $R$ are both planar:

- Theorem 4. Let $\Delta$ be a fixed constant, and let $H=H(n, \Delta)$ be a connected graph with $n$ vertices and maximum degree at most $\Delta$. Let $k=k(n) \rightarrow \infty$ as $n \rightarrow \infty$, and let $R=R(n, k)$ be a random graph on $V(H)$ consisting of exactly $k$ edges chosen uniformly at random from $\left(\begin{array}{c}V(H) \\ 2\end{array}\right)$. Let $G=G(n, \Delta, k)=H \cup R$. Then with high probability

$$
g(G)=\Theta(\max \{g(H), k\})
$$

The proof of Theorem 4 exploits a result from [10] for decomposing the base graph $H$ into various pieces. We construct a particular minor of $G$ where each of these pieces is condensed into a vertex (note that the genus of $G$ is at least the genus of any of its minors), and we find that we can obtain our result by applying Theorem 2 to this minor.

The remainder of the paper is structured as follows: in Section 2, we state the relevant terminology, notation, and key facts; in Section 3 , we focus on $g(n, m)$, proving Theorem 1 and providing sketch-proofs of Theorem 2 and Theorem 3; in Section 4, we present the fragile genus property, giving a sketch-proof of Theorem 4; and finally, in Section 5, we discuss the contiguity of $G(n)$ and $G(n, m)$ with random graph models of given genus. 


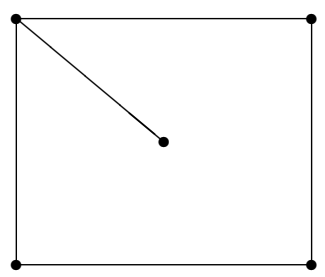

Figure 1 An embedding with faces of length six and four.

\section{Preliminaries and notation}

In this section, we provide details of the notation and definitions that will be used throughout, and then also present two results concerning the equivalence of $G_{n, p}$ and $G(n, m)$.

Let us first note that we shall always take $n$ and $m=m(n)$ to be integers satisfying $n>0$ and $m \geq 0$, even if this is not always explicitly stated.

We start with the definitions of the standard random graph models:

- Definition 5. We shall let $G(n, m)$ denote a graph taken uniformly at random from the set of all labelled graphs on the vertex set $[n]:=\{1,2, \ldots, n\}$ with exactly $m$ edges.

We shall let $G_{n, p}$ denote a graph on $[n]$ where every edge occurs independently at random with probability $p$, and we shall use $G(n)$ to denote $G_{n, \frac{1}{2}}$ (i.e. a graph taken uniformly at random from the set of all labelled graphs on $[n])$.

Next, we state the notation to be used for various key characteristics:

- Definition 6. Given a graph $G$, we shall use $g(G)$ to denote the genus of $G, \kappa(G)$ to denote the number of components of $G$, and $f(G)$ to denote the number of faces of $G$ when embedded on a surface of genus $g(G)$.

We also define $g(n, m):=g(G(n, m)), \kappa(n, m):=\kappa(G(n, m))$, and $f(n, m):=f(G(n, m))$.

Given a particular embedding of a graph, we shall use the length of a face to mean the number of edges with a side in the face, counting an edge twice if both sides are in the face (for example, the embedding shown in Figure 1 has one face of length 6 and one face of length 4).

We now also provide details of our order notation:

- Definition 7. Given non-negative functions $a(n)$ and $b(n)$, we shall use the following notation:

- $a(n)=\Omega(b(n))$ means there exists a constant $c>0$ such that $a(n) \geq c b(n)$ for all large $n$;

- $a(n)=O(b(n))$ means there exists a constant $C$ such that $a(n) \leq C b(n)$ for all large $n$;

- $a(n)=\Theta(b(n))$ means $a(n)=\Omega(b(n))$ and $a(n)=O(b(n))$;

- $a(n)=\omega(b(n))$ or $a(n) \gg b(n)$ means that, given any constant $K$, we have $\frac{a(n)}{b(n)}>K$ for all large $n$ (i.e. $\frac{a(n)}{b(n)} \rightarrow \infty$ as $\left.n \rightarrow \infty\right)$;

- $a(n)=o(b(n))$ or $a(n) \ll b(n)$ means that, given any constant $\epsilon>0$, we have $\frac{a(n)}{b(n)}<\epsilon$ for all large $n$ (i.e. $\frac{a(n)}{b(n)} \rightarrow 0$ as $\left.n \rightarrow \infty\right)$;

- $a(n) \sim b(n)$ means $a(n)=(1+o(1)) b(n)$.

We shall say that a random event $X_{n}$ happens with high probability (whp) if $\mathbb{P}\left(X_{n}\right) \rightarrow 1$ as $n \rightarrow \infty$. Given a non-negative random variable $a(n)$ and a non-negative function $b(n)$, we adapt the above deterministic definitions by replacing 'for all large $n$ ' with 'whp'. For 
example, $a(n)=o(b(n))$ whp or $a(n) \ll b(n)$ whp means that, given any constant $\epsilon>0$, we have $\frac{a(n)}{b(n)}<\epsilon$ whp.

We shall always take all asymptotics to be as $n \rightarrow \infty$, even if this is not always explicitly stated.

We shall shortly present the two aforementioned equivalence results for $G_{n, p}$ and $G(n, m)$. However, we first require the following definition:

- Definition 8. We say that a property is monotone increasing if whenever an edge is added to a graph with the property, then the resulting graph also has the property. Similarly, we say that a property is monotone decreasing if whenever an edge is deleted from a graph with the property, then the resulting graph also has the property. We say that a property is monotone if it is either monotone increasing or monotone decreasing.

We may now state the main equivalence result:

- Theorem 9 (see, for example, Proposition 1.15 of [6]). Given $m=m(n)$, let $p=p(n)=\frac{m}{\left(\begin{array}{c}n \\ 2\end{array}\right)}$. Then if a monotone property holds whp for $G_{n, p}$, it also holds whp for $G(n, m)$.

Note that, for any function $x=x(n)$, the property that $g(G) \leq x$ is monotone, as is the property that $g(G) \geq x$.

Unfortunately, the same cannot be said if we replace $g(G)$ with $f(G)$, the number of faces of $G$ when embedded on a surface of minimal genus. For instance, let $C_{4}^{+}$denote the graph formed be adding one edge to $C_{4}$, let $K_{5}^{-}$denote the graph formed be removing one edge from $K_{5}$, and note that we have $f\left(C_{4}\right)=2, f\left(C_{4}^{+}\right)=3, f\left(K_{5}^{-}\right)=6$, and $f\left(K_{5}\right)=5$ (observe that the first three graphs are planar, while $K_{5}$ has genus one). Hence, adding an edge can actually increase or decrease (or have no impact on) $f(G)$.

However, the function $g(G)-e(G)$ is certainly monotone decreasing (one way to see this is to note that Euler's formula gives $f(G)-e(G)=\kappa(G)+1-|G|-2 g(G)$, and $\kappa(G)$ and $g(G)$ are clearly monotone decreasing and monotone increasing, respectively). Using this, we may in fact still apply Theorem 9 to derive a useful equivalence result for the number of faces:

- Corollary 10. Let $m=m(n) \rightarrow \infty$ as $n \rightarrow \infty$, let $p=p(n)=\frac{m}{\left(\begin{array}{c}n \\ 2\end{array}\right)}$, and suppose $x=x(n)$ is a function such that $f\left(G_{n, p}\right) \leq x$ whp. Then

$$
f(n, m) \leq x+o(m) w h p .
$$

Proof. We are required to show that, given any constant $\epsilon>0$, we have $f(n, m)<x+\epsilon m$ whp.

Note that $e\left(G_{n, p}\right)$ has variance $\left(\begin{array}{l}n \\ 2\end{array}\right) p(1-p) \leq m$, and hence has standard deviation at most $m^{1 / 2}$, which is $o(m)$ since $m \rightarrow \infty$. Thus, since $e\left(G_{n, p}\right)$ has expectation exactly $m$, it follows that, given any constant $\epsilon>0$, we have $e\left(G_{n, p}\right)>(1-\epsilon) m$ whp. Therefore, since $f\left(G_{n, p}\right) \leq x$ whp, we then have $f\left(G_{n, p}\right)-e\left(G_{n, p}\right)<x-(1-\epsilon) m$ whp.

Now recall our observation that $f(G)-e(G)$ is a monotone decreasing function, from which it follows that the property that a graph satisfies $f(G)-e(G)<x-(1-\epsilon) m$ is monotone increasing. Hence, we may apply Theorem 9, thus obtaining $f(n, m)-m<x-(1-\epsilon) m$ whp, i.e. $f(n, m)<x+\epsilon m$ whp, as desired. 


\section{The genus of $G(n, m)$}

In this section, we provide an illustration of some of the techniques employed in the exploration of $g(n, m)$ by proving Theorem 1 , which will involve first obtaining bounds on $f(n, m)$ in Lemma 11 and Corollary 12. We then also give sketch-proofs of Theorem 2 and Theorem 3.

Lemma 11. Let $m=m(n)$ satisfy both $m \rightarrow \infty$ as $n \rightarrow \infty$ and $m \ll n^{1+\frac{1}{j}}$ for some fixed $j \in \mathbb{N}$. Then

$$
f(n, m) \leq(1+o(1)) \frac{2}{j+2} m w h p .
$$

Proof. We will use the $G_{n, p}$ model with $p=\frac{m}{\left(\begin{array}{c}n \\ 2\end{array}\right)}$, and show that the number of faces is at most $(1+o(1)) \frac{2}{j+2}\left(\begin{array}{c}n \\ 2\end{array}\right) p$ whp (we will then be done, by an application of Corollary 10). Thus, we are required to show that, given any constant $\epsilon>0$, the number of faces is at most $(1+\epsilon) \frac{2}{j+2}\left(\begin{array}{c}n \\ 2\end{array}\right) p$ whp.

We will follow a similar argument to that used in the proof of Theorem 1.2 of [16], which involves showing that whp $G_{n, p}$ will have few short cycles, and hence few small faces, and hence few faces in total.

Note that the expected number of cycles in $G=G_{n, p}$ of length at most $j+1$ is

$$
\begin{aligned}
\sum_{i=1}^{j+1}\left(\begin{array}{c}
n \\
i
\end{array}\right) \frac{i !}{2 i} p^{i} & \leq \sum_{i=1}^{j+1} \frac{n^{i} p^{i}}{2 i} \leq \sum_{i=1}^{j+1}(n p)^{i} \\
& \left.\leq(j+1) \max \left\{n p,(n p)^{j+1}\right\} \quad \text { (since either } n p \leq 1 \text { or } n p \geq 1\right) \\
& =O\left(\max \left\{n p,(n p)^{j+1}\right\}\right) \\
& =O\left((n p) \max \left\{1,(n p)^{j}\right\}\right) \\
& =o\left(n^{2} p\right) \quad\left(\text { since } 1 \ll n \text { and } n p=\frac{n m}{\left(\begin{array}{c}
n \\
2
\end{array}\right)} \ll \frac{n^{2+\frac{1}{j}}}{n^{2}}=n^{\frac{1}{j}}\right) .
\end{aligned}
$$

Thus, by Markov's inequality, we can say that whp $G$ has no more than $\frac{1}{2(j+2)} \epsilon\left(\begin{array}{l}n \\ 2\end{array}\right) p$ cycles of length at most $j+1$.

Let us now consider an embedding of $G$. Note that the statement of this lemma is certainly true if $G$ is acyclic (since then there is only one face), so we may assume that $G$ is not acyclic, in which case every face of the embedding must contain a cycle.

Let $f^{\prime}$ denote the number of faces in this embedding with length at most $j+1$. Then every such face must contain a cycle of length at most $j+1$, and every such cycle can only be included in at most two faces. Hence, whp we have

$$
f^{\prime} \leq \frac{1}{j+2} \epsilon\left(\begin{array}{l}
n \\
2
\end{array}\right) p
$$

Now let $f$ denote the total number of faces in this embedding, and observe that

$2 e(G) \geq 3 f^{\prime}+(j+2)\left(f-f^{\prime}\right)=(j+2) f-(j-1) f^{\prime}$. 
Thus, we have

$$
\begin{aligned}
f & \leq \frac{2}{j+2} e(G)+\frac{j-1}{j+2} f^{\prime} \leq \frac{2}{j+2} e(G)+f^{\prime} \\
& \leq \frac{2}{j+2} e(G)+\frac{1}{j+2} \epsilon\left(\begin{array}{l}
n \\
2
\end{array}\right) p \quad \text { whp by (2) } \\
& \leq \frac{2}{j+2}\left(1+\frac{\epsilon}{2}\right)\left(\begin{array}{l}
n \\
2
\end{array}\right) p+\frac{2}{j+2} \frac{\epsilon}{2}\left(\begin{array}{l}
n \\
2
\end{array}\right) p \quad \text { whp } \\
& =(1+\epsilon) \frac{2}{j+2}\left(\begin{array}{l}
n \\
2
\end{array}\right) p
\end{aligned}
$$

and so we are done.

We now obtain our aforementioned corollary, which gives a useful bound on the number of faces $f(n, m)$ when $m \leq n^{1+o(1)}$ :

- Corollary 12. Let $m=m(n)$ satisfy both $m \rightarrow \infty$ as $n \rightarrow \infty$ and $m \leq n^{1+o(1)}$. Then

$$
f(n, m)=o(m) w h p
$$

Proof. We are required to show that, given any constant $\epsilon>0$, we have $f(n, m)<\epsilon m$ whp.

We may simply choose a value $j \in \mathbb{N}$ such that $j>\frac{2(1+\epsilon)}{\epsilon}-2$, in which case $\frac{2}{j+2}<\frac{\epsilon}{1+\epsilon}$. Then, by Lemma 11, we have

$$
\begin{aligned}
f(n, m) & <(1+\epsilon) \frac{2}{j+2} m \text { whp } \\
& <(1+\epsilon) \frac{\epsilon}{1+\epsilon} m \\
& =\epsilon m
\end{aligned}
$$

and so we are done.

We may now easily derive Theorem 1:

Proof of Theorem 1. The upper bound holds for all $m$-we simply use Euler's formula

$$
g(n, m)=\frac{1}{2}(m-n-f(n, m)+\kappa(n, m)+1)
$$

from (1), and observe that $n \geq \kappa(n, m)$ and $f(n, m) \geq 1$.

The lower bound also follows from Euler's formula, using $n=o(m)$ and $f(n, m)=o(m)$ whp by Corollary 12 .

Theorem 2 is obtained similarly:

Sketch of Proof of Theorem 2. The proof again utilises Euler's formula and Corollary 12. For Theorem 1, the role of the number of components $\kappa(n, m)$ was insignificant, since we had $m \gg n \geq \kappa(n, m)$. However, since we now have $m=O(n)$, this time we find that we do require accurate information on $\kappa(n, m)$, and we extract this from Theorem 6.12 of [3].

The proof of Theorem 3 is more intricate: 


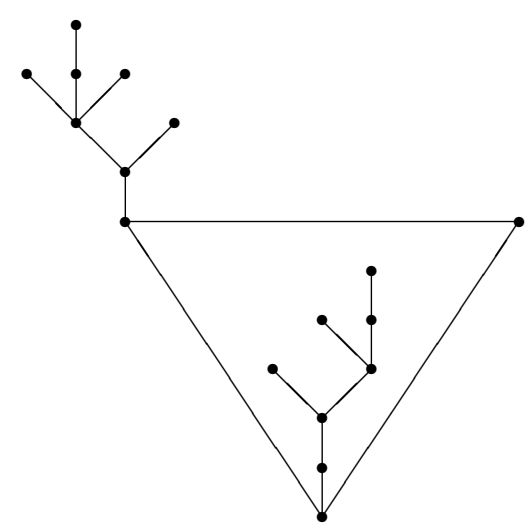

Figure 2 An embedding with two large faces.

Sketch of Proof of Theorem 3. Recall that the proof of Lemma 11 involved bounding the number of small faces via calculations on the number of short cycles. Clearly, this may greatly over-estimate the number of small faces if there are actually many large faces that consist of a short cycle with large trees rooted on the cycle (see Figure 2).

Consequently, in order to achieve the desired level of accuracy for Theorem 3 , much of the proof will this time involve working directly with the 2-core of the giant component instead of with the entire graph (note that whp this determines the overall genus, and also alleviates any need to compute the number of components).

Thus, we shall proceed towards an application of Euler's formula for the 2-core of the giant component, which will involve us first needing to establish bounds for the number of faces in the 2-core of the giant.

Our strategy here is to begin by examining the number of 'short' cycles in $G(n, m)$ that also satisfy some additional properties; then to use this to bound the number of such cycles in the giant component; then (by careful consideration of the additional properties) to bound the total number of short cycles in the giant component (and hence in the 2-core of the giant); then to use this to bound the number of short faces in the 2-core of the giant; and then, finally, to separately bound the number of large faces in the 2-core of the giant (via the number of edges).

We start by utilising work from [13] on cycles in $G(n, m)$. For our region when $m=\frac{n}{2}+s$ for $s>0$ satisfying $n^{2 / 3} \ll s \ll n$, results are given here concerning the number of cycles in $G(n, m)$ that both (a) have length at most $\frac{i n}{s}$, for fixed $i \in \mathbb{N}$, and (b) satisfy certain technical properties involving the neighbouring vertices and the trees rooted on the cycles.

In particular, it is shown that the number of these cycles tends in probability to a random variable that has a Poisson distribution with mean $\lambda(i)$, for a given monotonically increasing function $\lambda$, and hence that the number of such cycles is concentrated around $\lambda(i)$.

We extend this latter result to cover the case when $i(n)$ is a function of $n$, as long as $i(n)$ grows sufficiently slowly.

Next, we construct a specific function $i(n)$ with $i(n) \rightarrow \infty$ for which $\lambda(i(n))$ also grows very slowly. For this function, we manage to show that the number of cycles in $G(n, m)$ of length at most $\frac{i(n) n}{s}$ with the aforementioned technical properties is still only $o\left(\frac{s^{3}}{n^{2}}\right)$ whp. The key factor here is that $s^{3} \gg n^{2}$.

We then move to the giant component of $G(n, m)$. By utilising further useful results from [13], we are able to show that whp all cycles of length at most $\frac{i(n) n}{s}$ in the giant component will actually satisfy all of the various technical properties. 
Thus, we find that the total number of cycles of length at most $\frac{i(n) n}{s}$ in the giant component of $G(n, m)$ (or, equivalently, in the 2-core of the giant component) must be $o\left(\frac{s^{3}}{n^{2}}\right)$ whp. Hence, the number of faces of length at most $\frac{i(n) n}{s}$ in the 2-core of the giant must be $o\left(\frac{s^{3}}{n^{2}}\right)$ whp too.

We then consider the number of faces of length at least $\frac{i(n) n}{s}$ in the 2-core of the giant. By results from [12] and [13], the number of edges in the 2-core of the giant is known to be $\Theta\left(\frac{s^{2}}{n}\right)$ whp, and so the number of such faces can only be $O\left(\frac{s^{3}}{i(n) n^{2}}\right)$ whp. Crucially, our earlier work to ensure that $i(n) \rightarrow \infty$ then implies that this is $o\left(\frac{s^{3}}{n^{2}}\right)$.

Hence, putting everything together, we find that the total number of faces in the 2-core of the giant is also $o\left(\frac{s^{3}}{n^{2}}\right)$ whp.

We then finish with an appropriate application of Euler's formula, using existing results from [12] and [13] on the number of vertices and edges in the 2-core of the giant. Note that our bound for the number of faces is sufficiently precise to achieve the desired level of accuracy.

\section{$4 \quad$ The fragile genus property}

In this section, we provide a sketch-proof of Theorem 4, which shows that the genus of any given connected graph with bounded degree can increase dramatically if a small number of random edges are added.

Sketch of Proof of Theorem 4. Note that adding an edge can only increase the genus by at most one, so we certainly have $g(G) \leq g(H)+k \leq 2 \max \{g(H), k\}$. Also, we clearly have $g(G) \geq g(H)$. Hence, it just remains to show that $g(G) \geq \Omega(k)$ whp.

The result for $\lim _{\inf } \ln _{n \rightarrow \infty} \frac{k}{n}>\frac{1}{2}$ can be obtained simply by applying our results on the genus of $G(n, m)$ to $R$ (with $m=k$ ), and so we may assume that $k \leq n$, say.

Our proof involves contracting carefully chosen identically-sized pieces of the graph (we use 'piece' to mean a connected subgraph) into 'super-vertices' - note that this cannot increase the genus. We then show that the uniform random graph induced by these super-vertices and the random edges will whp be sufficiently dense for us to be able to apply Theorem 2 .

We start by splitting the base graph $H$ into $t=\Theta(k)$ connected pieces $V_{1}, V_{2}, \ldots, V_{t}$ (hence our earlier assumption that $k=O(n)$ ), plus a few $(o(n))$ vertices that do not belong to any of these pieces.

Using a decomposition result from [10], we may select the pieces in such a way that we have

$$
\frac{5 n \Delta^{2}}{k} \leq\left|V_{i}\right| \leq \frac{10 n \Delta^{3}}{k}
$$

for all $i$, and so

$$
(1-o(1)) \frac{k}{10 \Delta^{3}} \leq t \leq \frac{k}{5 \Delta^{2}} .
$$

Let us note that the value of $t$ has been carefully arranged here. It will be crucial for our later calculations that we have $t=\Omega(k)$, but also that $\frac{t}{k}$ is not too large.

As mentioned, we shall wish to condense pieces of our graph into super-vertices. However, rather than contracting the entire pieces $V_{1}, V_{2}, \ldots, V_{t}$, for each $i$ we instead select a connected subpiece $U_{i} \subset V_{i}$ with size $\left|U_{i}\right|=s:=\min _{i}\left|V_{i}\right|$. This will be important to ensure uniformity, so that we can later apply Theorem 2 . 


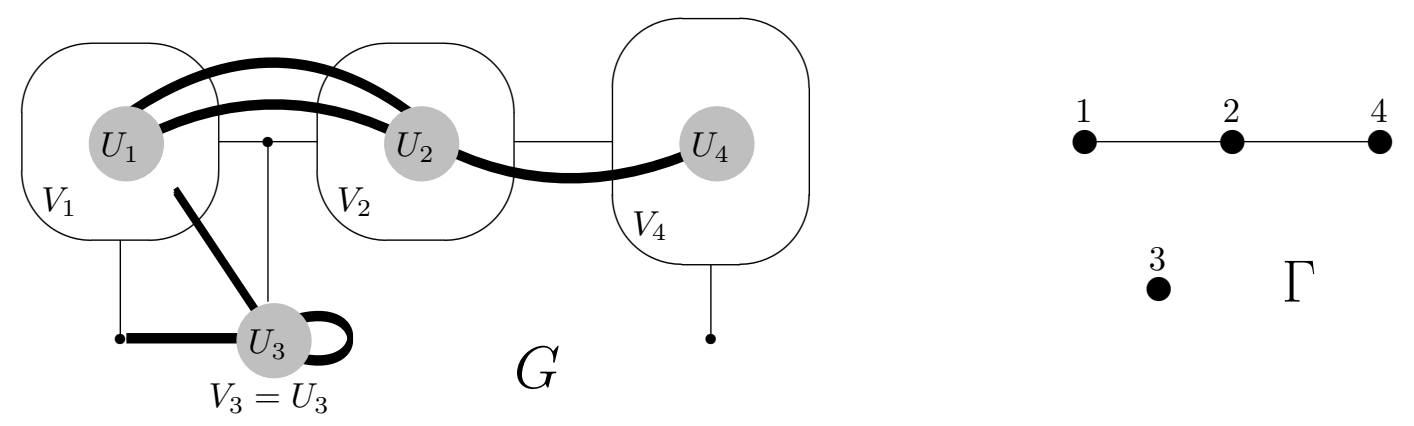

Figure 3 A graph $G$ and the corresponding graph $\Gamma$.

We then contract each of these subpieces $U_{1}, U_{2}, \ldots, U_{t}$, and ignore any vertices not in these subpieces. Formally, this means that we define an auxiliary random graph $\Gamma$ with vertex set $[t]$, where two vertices $i, j \in[t]$ are connected by an edge if and only if there is an edge of $R$ going between $U_{i}$ and $U_{j}$ (see Figure 3, where thick lines denote the edges of $R$ - note in particular that this example has no edge in $\Gamma$ between vertex 1 and vertex 3 , as there is no edge in $G$ between $U_{1}$ and $U_{3}$ ).

Observe that $\Gamma$ is a minor of $G$, and hence that $g(G) \geq g(\Gamma)$. Thus, since we deliberately chose $t$ to be large enough that $t=\Omega(k)$ (recall (4)), it will suffice to show that $g(\Gamma)=\Omega(t)$ whp.

Note that the number of edges in $\Gamma$ is not equal to $k$, since we only include edges between our chosen subpieces, and we only include at most one edge for each such pair $U_{i}, U_{j}$.

In order to obtain a bound for $e(\Gamma)$, we consider the edges of $R$ one-by-one (in a random order). Note that $e(\Gamma)$ is then precisely equal to the number of edges of $R$ which satisfy the two properties that

- (a) the edge lies between a vertex of $U_{i}$ and a vertex of $U_{j}$ for $i \neq j$;

- and (b) no previous edges of $R$ lie between these same two sets $U_{i}$ and $U_{j}$.

Observe that the probability that an edge of $R$ satisfies both (a) and (b) is always at least

$$
\frac{\left(\left(\begin{array}{l}
t \\
2
\end{array}\right)-(k-1)\right) s^{2}}{\left(\begin{array}{l}
n \\
2
\end{array}\right)},
$$

since there are at least $\left(\begin{array}{l}t \\ 2\end{array}\right)-(k-1)$ ways to choose a pair $U_{i}, U_{j}$ which do not already have an edge of $R$ between them, and then $s$ ways to choose a vertex from $U_{i}$, and $s$ ways to choose a vertex from $U_{j}$.

By (3) and (4), this probability is at least $(1-o(1)) \frac{1}{4 \Delta^{2}}$, and so we can certainly say that whp at least $\frac{k}{5 \Delta^{2}}$ of the $k$ edges of $R$ will satisfy conditions (a) and (b). Thus, $e(\Gamma) \geq \frac{k}{5 \Delta^{2}}$ whp.

Crucially, the fact that we chose $t$ so that $t \leq \frac{k}{5 \Delta^{2}}$ (recall (4)) consequently means that we have $e(\Gamma) \geq t$ whp.

We then let $\Gamma^{*}$ be the random graph formed by considering just $t$ (randomly chosen) edges of $\Gamma$. Since each set $U_{i}$ had exactly the same number of vertices, this graph $\Gamma^{*}$ is in fact a uniform random graph with $t$ vertices and $t$ edges. Thus, by Theorem 2 , we have $g\left(\Gamma^{*}\right)=\Theta(t)$ whp, and so $g(\Gamma)=\Omega(t)$ whp, as required.

Note that Theorem 4 implies the remarkable fact that whp $G=H \cup R$ will have $\Omega(n)$ genus even if $H$ is a planar graph and $k=\epsilon n$ for some very small (but positive) $\epsilon$ ! We thus call this the 'fragile genus' property. 
Let us conclude this section by also remarking that for $\lim \sup _{n \rightarrow \infty} \frac{k}{n}<\frac{1}{2}$, the restriction on the maximum degree in Theorem 4 is essential, since otherwise we could take $H$ to be a star (note that whp the random graph $R$ would consist only of trees and unicyclic components, and would consequently be outerplanar, and so the overall graph $G$ would then have genus zero).

\section{Discussion}

As mentioned in the introduction, one of our motivations for studying the genus of $G(n, m)$ comes from recent work on random graphs on surfaces. In particular, one may define $S_{g}(n)$ to be a graph taken uniformly at random from the set of all labelled graphs on $[n]$ with genus at most $g$, and $S_{g}(n, m)$ to be a graph taken uniformly at random from the set of all labelled graphs on $[n]$ with exactly $m$ edges and with genus at most $g$. It is then natural to ask when these graphs will be contiguous with $G(n)$ and $G(n, m)$.

It immediately follows from the work in [1] that $G(n)$ and $S_{g}(n)$ are certainly contiguous for any $g(n)$ satisfying $g(n) \geq(1+\epsilon) \frac{n^{2}}{24}$ for any $\epsilon>0$ (and also for some $g(n)$ satisfying $\left.g(n)=(1+o(1)) \frac{n^{2}}{24}\right)$, since $G(n)$ will have genus at most $(1+o(1)) \frac{n^{2}}{24}$ whp.

Conversely, $G(n)$ and $S_{g}(n)$ are certainly not contiguous for any $g(n)$ satisfying $g(n) \leq$ $(1-\epsilon) \frac{n^{2}}{24}$ for any $\epsilon>0$ (and also not for some $g(n)$ satisfying $\left.g(n)=(1+o(1)) \frac{n^{2}}{24}\right)$, since there is then a discrepancy with respect to the property of having genus greater than $g$ (note $\mathbb{P}\left[S_{g}(n)\right.$ has genus $\left.>g\right]=0$, by definition, but $\mathbb{P}[G(n)$ has genus $>g] \rightarrow 1$ as $\left.n \rightarrow \infty\right)$.

By the same arguments, similarly precise results for the contiguity of $G(n, m)$ and $S_{g}(n, m)$ for the various different regions of $m$ can now also be obtained.

\section{References}

1 A. Archdeacon and D. Grable. The genus of a random graph. Discrete Math., 142:21-37, 1995 .

2 T. Bohman, A. Frieze, and R. Martin. How many random edges make a dense graph hamiltonian? Random Struct. Algor., 22:33-42, 2003.

3 B. Bollobás. Random Graphs. Cambridge University Press, Cambridge, 2001.

4 C. Dowden, M. Kang, and P. Sprüssel. The evolution of random graphs on surfaces. SIAM J. Discrete Math., 32:695-727, 2018.

5 A. Frieze and M. Karoński. Introduction to Random Graphs. Cambridge University Press, Cambridge, 2015.

6 S. Janson, T. Łuczak, and A. Ruciński. Random Graphs. Wiley, New York, 2000.

7 M. Kang, M. Moßhammer, and P. Sprüssel. Phase transitions in graphs on orientable surfaces. submitted, arXiv:1708.07671.

8 M. Krivelevich, M. Kwan, and B. Sudakov. Cycles and matchings in randomly perturbed digraphs and hypergraphs. Combin. Probab. Comput., 25:909-927, 2016.

9 M. Krivelevich, M. Kwan, and B. Sudakov. Bounded-degree spanning trees in randomly perturbed graphs. SIAM J. Discrete Math., 31:155-171, 2017.

10 M. Krivelevich and A. Nachmias. Colouring complete bipartite graphs from random lists. Random Struct. Algor., 29:436-449, 2006.

11 M. Krivelevich, D. Reichman, and W. Samotij. Smoothed analysis on connected graphs. SIAM J. Discrete Math., 29:1654-1669, 2015.

12 T. Euczak. Component behaviour near the critical point of the random graph process. Random Struct. Algor., 1:287-310, 1990.

13 T. Łuczak. Cycles in a random graph near the critical point. Random Struct. Algor., 2:421-439, 1991. 
14 T. Łuczak, B. Pittel, and J. C. Wierman. The structure of a random graph near the point of the phase transition. Trans. Amer. Math. Soc., 341:721-748, 1994.

15 M. Noy, V. Ravelomanana, and J. Rué. The probability of planarity of a random graph near the critical point. Proc. Amer. Math. Soc., 143:925-936, 2015.

16 V. Rödl and R. Thomas. On the genus of a random graph. Random Struct. Algor., 6:1-12, 1995. 